\title{
Integrating Process Management and Content Management for Service Industries
}

\author{
Young Gil Kim, Chul Young Kim, and Sang Chan Park \\ Dept. of Industrial Engineering, Korea Advanced Institute of Science and Technology \\ (KAIST), 373-1 Gusung-dong, Yusung-gu, Daejeon, Korea \\ \{ttaldul, fezero, sangchanpark\}@kaist.ac.kr
}

\begin{abstract}
Content management utilizing Web technologies plays an important role in e-business environment because it enables the seamless flow of information among business participants. In this paper, we present a framework for incorporating content management facilities into a process management system and apply to marketing research processes for demonstrating the feasibility of the proposed framework.
\end{abstract}

\section{Introduction}

Competitive pressures of the modern global economy are forcing business participants to continually improve their performances such as quality, speed and cost. Also today's management trends shift from data-centric to process-centric approach [1]. New technologies have become available for adopting this approach. However business executives have struggled to justify continued investments into the systems to reflect the increasing knowledge intensity of all types of work in the organization.

\section{Related Works}

Process management systems can be used to integrate existing applications and support process change by merely changing the process diagram. Web and Internet tools can support these aspects effectively. Content management (CM) is generally a term that describes the issues around creating, versioning, storing and disseminating information [2], or it is often equated a repository based facility to store contents with some metadata management. While most researches are focused on content management to manage and integrate content-flow itself, we concentrate on content management supporting to process management within organization's workflows.

\section{Framework and Implementation}

To construct a process management system incorporated with CM facilities, it should be identified firstly what process-related contents is. As depicted in Fig. 1 left, we define process-related contents as that applied in a process. These contents would be bound with XML metadata and stored in repositories. The elements for the metadata consist of three major elements: organization, description, and deployment. 

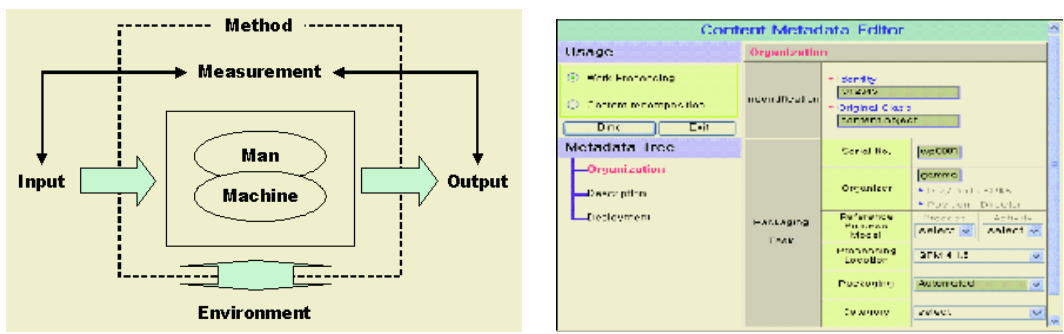

Fig. 1. The entities of process contents and the web interface for content metadata template

For streamlined and effective supports for building mature processes, our idea is to embed the structure of information items into metadata template, which are then filled on the fly doing daily work. The contents used in or produced from multiple sources are registered with automated metadata capturing facilities (See Fig. 1 right).

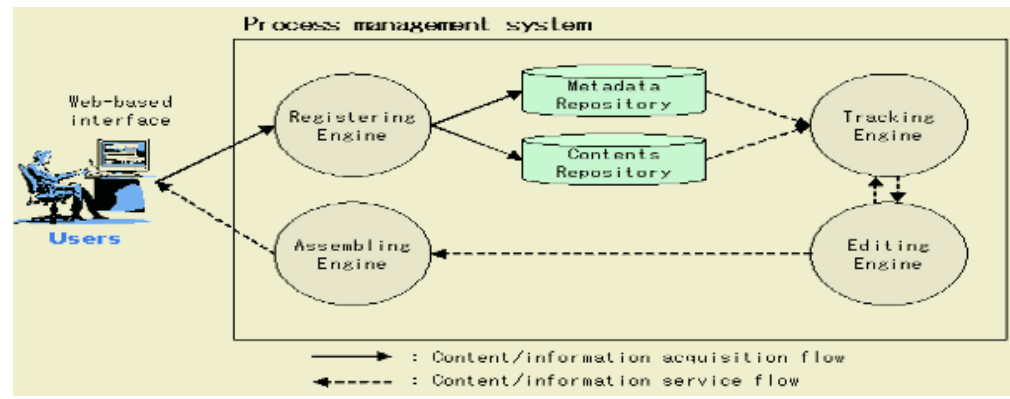

Fig. 2. The framework for content management engines incorporating into process management

The proposed framework has four major engines and two repositories for managing process-related contents (See Fig. 2). The primary role of the CM modules incorporated in a process management system is to bind contents with synthesized metadata and so, to acquire, manage, reused, and service the various types of contents that are produced from a process. The role of each component in process management is:

- Registering engine: bind contents with the metadata using XML and register contents and metadata to the repositories. The metadata is whether deployment and processing information of the registered contents or content structure itself;

- Tracking engine: with seeking out metadata, retrieval and extract the appropriate contents required by users;

- Editing engine: Edit and organize the constitutions of contents, that is, this engine can make various contents needed to a content constitution organized to hierarchical structure or re-composite contents;

- Assembling engine: assemble contents using the content constitution information produced by editing engine for visualizing contents;

- Metadata repository: store metadata elements using XML and database, also it includes the information of prior content constitutions;

- Content repository: store content objects. It can be physical repository or electrical database. It has the information about a physical location of contents. 

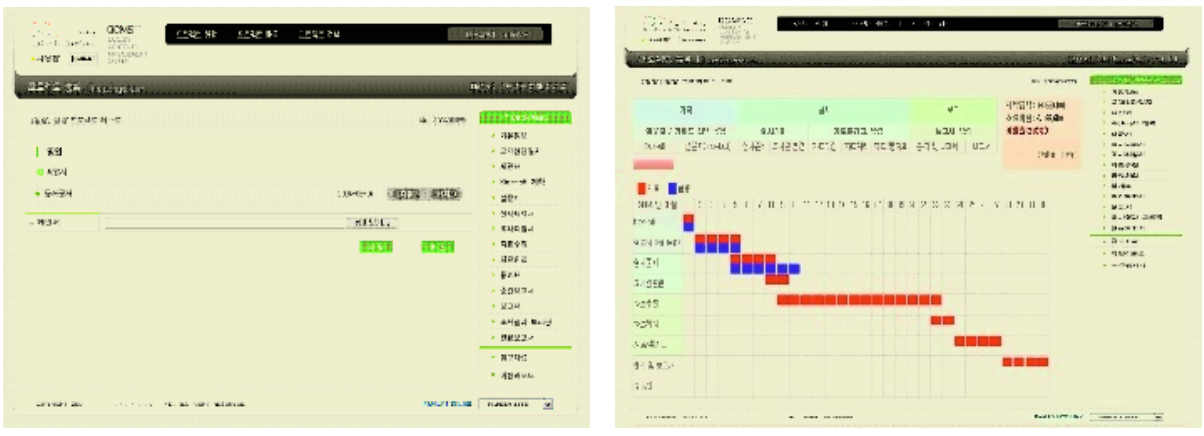

Fig. 3. Snapshots of registering and monitoring interface for a marketing research process

In implementation, a content produced from a process activity is stored in the content repository through the registering engine. In that time, the content is bound and packaged with XML-based metadata having three major part collected by metadata editor, and the metadata is stored in the metadata repository. The stored object content is packaged with other contents produced in the activity according to the organization part of metadata (See Fig. 1 right and Fig. 3 left). Fig. 3 right shows monitoring interface for certain marketing research project. This page includes the key contents of critical processes of the project and shows the project status according to a predefined content structure and deployment information using the $\mathrm{CM}$ engines.

\section{Conclusion}

In this paper, we presented a methodology for developing CM facility-based process management system. The metadata including process entities with $4 \mathrm{M} 1 \mathrm{E}$ and content aggregation methods could be used for better understanding a company's processes and easily constructing a web-based process management system. Also the process content metadata consisting of organization, description, and deployment parts could be used for process control and content recomposing and reuse. The proposed framework enables real-time content integration among user's workflow information, organizational knowledge, and a variety of business applications.

\section{References}

1. van der Aalst, W.M.P.: Business Process Management: A personal view. BPMJ, Vol. 10. No. 2 (2004) 248-253

2. Stonebraker, M., Hellerstein, J. M.: Content Integration for E-Business. ACM SIGMOD Record, Vol. 30, Issue 2 (2001) 552-560 